\title{
CORRIGENDUM
}

\section{Cloning and characterization of a tryptophanase gene from Enterobacter aerogenes SM-18}

Kosei Kawasaki, Atsushi Yokota, Shigeru Oita, Chisa Kobayashi, Shuji Yoshikawa, Shin-Ichi Kawamoto, Shoichi Takao and Fusao Tomita

Journal of General Microbiology (1993), 139, 3275-3281.

Table 1, line 1: Yokota \& Takao (1984) should read Yokota \& Takao (1989)

p. 3280, line 15: (Phe-Asn-Ile-Glu; ... should read (Phe-Asn-Ile-Asp; ... 